\title{
CHEBYSHEV TYPE ESTIMATES FOR BEURLING GENERALIZED PRIME NUMBERS. II
}

\author{
WEN-BIN ZHANG
}

Abstract. Let $N(x)$ be the distribution function of the integers in a Beurling generalized prime system. The Chebyshev type estimates for Beurling generalized prime numbers in the general case

$$
N(x)=x \sum_{\nu=1}^{n} A_{\nu} \log ^{\rho_{\nu}-1} x+O\left(x \log ^{-\gamma} x\right)
$$

is a long standing question. In this paper we shall give an affirmative answer to the question by proving that the Chebyshev type estimates

$$
0<\liminf _{x \rightarrow \infty} \frac{\psi(x)}{x}, \quad \limsup _{x \rightarrow \infty} \frac{\psi(x)}{x}<\infty
$$

hold even under weaker condition

$$
\int_{1}^{\infty} x^{-1}\left\{\sup _{x<y} y^{-1}\left|N(y)-y \sum_{\nu=1}^{n} A_{\nu} \log ^{\rho_{\nu}-1} y\right|\right\} d x<\infty
$$

with $\rho_{n}=\tau \geq 1,0<\rho_{1}<\rho_{2}<\cdots<\rho_{n}$, and $A_{n}>0$. This generalizes a result of Diamond and a result of the present author.

\section{INTRODUCTION}

Let $\psi(x)$ be the weighted counting function of the ordinary prime numbers. Chebyshev was the first to establish the correct order of magnitude of $\psi(x)$ by showing that there exist two numbers $\alpha>0$ and $\beta<\infty$ such that

$$
\liminf _{x \rightarrow \infty} \frac{\psi(x)}{x} \geq \alpha, \quad \limsup _{x \rightarrow \infty} \frac{\psi(x)}{x} \leq \beta .
$$

The prime number theorem (P.N.T.) asserts that $\alpha=\beta=1$. In [4], Diamond established Chebyshev type estimates for Beurling generalized primes. Here we shall generalize Diamond's result.

Let $\mathscr{P}=\left\{p_{i}\right\}_{i=1}^{\infty}$, where $1<p_{1} \leq p_{2} \leq \ldots, p_{i} \rightarrow \infty$, be a set of Beurling generalized (henceforth, $g$-) prime numbers and $\mathscr{N}=\left\{N_{i}\right\}_{i=0}^{\infty}$ be the associated set of $g$-integers $($ see $[1,2])$. Define

$$
N(x)=\sum_{\substack{i \\ n_{i} \leq x}} 1, \quad \psi(x)=\sum_{\substack{i, \alpha \\ p_{i}^{\alpha} \leq x}} \log p_{i} .
$$

Received by the editors December 28, 1990.

1980 Mathematics Subject Classification (1985 Revision). Primary 11 N80.

Key words and phrases. Beurling generalized prime numbers, Chebyshev type estimates, approximate convolution inverse. 
Beurling [2] proved that if

$$
N(x)=A x+O\left(x \log ^{-\gamma} x\right)
$$

for some constants $A>0$ and $\gamma>3 / 2$, then the P.N.T. holds for $\mathscr{P}$. If $\gamma=3 / 2$ in (1.2), the P.N.T. need not hold as Diamond [4] showed by an example based on a continuous example of Beurling. Diamond [5] also showed that if $\gamma>1$ in (1.2) then (1.1) holds. On the other hand, (1.1) is not generally true if $\gamma<1$, as an example of Hall [7] shows.

Beurling investigated also the more general case in which

$$
N(x)=x \sum_{\nu=1}^{n} A_{\nu} \log ^{\rho_{\nu}-1} x+O\left(x \log ^{-\gamma} x\right),
$$

where $\rho_{1}<\rho_{2}<\cdots<\rho_{n}$ and $A_{1}, A_{2}, \ldots, A_{n}$ are arbitrary real numbers. He showed that if (1.3) with $1 \leq \rho_{n}=\tau<2$ holds for some $A_{n}>0$ and $\gamma>1+\tau / 2$ then $\lim _{x \rightarrow \infty} \psi(x) / x=\tau$, a generalization of the P.N.T. However, if $\tau \geq 2$, even an $O(1)$ error term in (1.3) does not guarantee $\psi(x) \sim \tau x$. Still, Beurling proved that if (1.3) holds with $\rho_{n}=\tau \geq 2$ for some $A_{n}>0$ and $\gamma>1+\tau / 2$ then there exist $0<t_{1} \leq t_{2} \leq \cdots \leq t_{q}<\infty$ with $q \leq[\tau / 2]$ such that

$$
\psi(x) \sim x\left\{\tau-2 \sum_{\nu=1}^{q} \cos \left(t_{\nu} \log x-\operatorname{arctg} t_{\nu}\right)\right\} .
$$

This gave rise to the long standing question of generalizing Chebyshev type estimates for the case (1.3). In the present paper, we shall prove the following theorem which gives an affirmative answer to the question.

Theorem. Let $0<\rho_{1}<\rho_{2}<\cdots<\rho_{n}$ and $A_{1}, A_{2}, \ldots, A_{n}$ be arbitrary real numbers. If

$$
\int_{1}^{\infty} x^{-1}\left\{\sup _{x \leq y} y^{-1}\left|N(y)-y \sum_{\nu=1}^{n} A_{\nu} \log ^{\rho_{\nu}-1} y\right|\right\} d x<\infty
$$

holds with $\rho_{n}=\tau \geq 1$ and $A_{n}=A>0$, then there exist numbers $\alpha>0$ and $\beta<\infty$ for which (1.1) holds.

This theorem is a generalization of the results in $[5,8]$. In particular, it has the following immediate consequence.

Corollary. If (1.3) holds with $\rho_{n}=\tau \geq 1$ for some $A_{n}=A>0$ and $\gamma>1$, then (1.1) is true.

The proof of the Theorem will utilize Beurling's asymptotic analysis [2], Diamond's approximate convolution inverse [5,6], and the author's idea in [8]. Elementary convolution techniques [3] play the main role in the proof. It would also be interesting to find an analytic proof.

We remark that (1.1) is not generally true if $\gamma<1$ in (1.3) as the example of Hall mentioned above shows.

\section{Preliminaries}

In the proof of the Theorem, we shall make frequent use of multiplicative convolution techniques which have been described in detail in [3]. In particular, we need the following preliminaries. 
Let $d t$ denote the Lebesgue measure on the Borel subsets of $[1, \infty)$. Let $\rho>0,0 \leq \varepsilon \leq 1$. We define

$$
\left(\delta-\varepsilon t^{-\varepsilon} d t\right)^{\rho}=\delta+\sum_{k=1}^{\infty} \frac{\rho(\rho-1) \cdots(\rho-k+1)}{k !}\left(-\varepsilon t^{-\varepsilon} d t\right)^{k},
$$

where $\delta$ represents point mass 1 at 1 . We note that the left-hand side is a noninteger convolution power if $\rho$ is not an integer. Hence,

$$
\begin{aligned}
& \left(\delta-\varepsilon t^{-\varepsilon} d t\right)^{\rho} \\
& \quad=\delta+\left(\sum_{k=1}^{\infty} \frac{\rho(\rho-1) \cdots(\rho-k+1)}{k !}(-\varepsilon)^{k} \frac{\log ^{k-1} t}{(k-1) !}\right) t^{-\varepsilon} d t .
\end{aligned}
$$

Similarly,

$$
\begin{aligned}
(\delta+ & \left.(1-\varepsilon) t^{-\varepsilon} d t\right)^{\rho} \\
& =\delta+\sum_{k=1}^{\infty} \frac{\rho(\rho-1) \cdots(\rho-k+1)}{k !}\left((1-\varepsilon) t^{-\varepsilon} d t\right)^{k} \\
& =\delta+\left(\sum_{k=1}^{\infty} \frac{\rho(\rho-1) \cdots(\rho-k+1)}{k !}(1-\varepsilon)^{k} \frac{\log ^{k-1} t}{(k-1) !}\right) t^{-\varepsilon} d t .
\end{aligned}
$$

We note that the sum on the right-hand side of (2.2) and (2.3) has only a finite number of terms and is easy to deal with if $\rho$ is a positive integer. In the discussion below, special analysis will be required if $\rho$ is not a positive integer.

By the exponential representation [3], it is easy to prove the following formulas

$$
\left(\delta-\varepsilon t^{-\varepsilon} d t\right)^{\rho}=\exp \left\{-\rho \frac{1-t^{-\varepsilon}}{\log t} d t\right\}
$$

and

$$
\left(\delta+(1-\varepsilon) t^{-\varepsilon} d t\right)^{\rho}=\exp \left\{\rho t^{-\varepsilon} \frac{1-t^{-(1-\varepsilon)}}{\log t} d t\right\}
$$

which are generalizations of $(3.2 \mathrm{~d})$ in [3]. Therefore,

$$
\begin{gathered}
\left(\delta-\varepsilon t^{-\varepsilon} d t\right)^{\rho_{1}+\rho_{2}}=\left(\delta-\varepsilon t^{-\varepsilon} d t\right)^{\rho_{1}} *\left(\delta-\varepsilon t^{-\varepsilon} d t\right)^{\rho_{2}}, \\
(\delta+d t)^{\rho} *\left(\delta-\varepsilon t^{-\varepsilon} d t\right)^{\rho}=\left(\delta+(1-\varepsilon) t^{-\varepsilon} d t\right)^{\rho},
\end{gathered}
$$

and

$$
(\delta+d t)^{\rho} *\left(\delta-t^{-1} d t\right)^{\rho}=\delta .
$$

Moreover, we have

$$
(\delta+d t)^{\rho} *\left(\delta-\varepsilon t^{-\varepsilon} d t\right)^{\tau}=\left(\delta+(1-\varepsilon) t^{-\varepsilon} d t\right)^{\rho} *\left(\delta-\varepsilon t^{-\varepsilon} d t\right)^{\tau-\rho}
$$

if $\tau>\rho$.

We then consider the Mellin transform

$$
\widehat{F}(s):=\int_{1}^{\infty} x^{-s}\left(\delta-\varepsilon x^{-\varepsilon} d x\right)^{\rho}
$$

(following [3], in this paper, an integral $\int_{x}^{y}$ means $\int_{x+}^{y+}$ except when $x=1$, in which case we take the lower limit to be 1-), which is convergent in the half-plane $\operatorname{Re} s=\sigma>1$ if $\rho$ is not a positive integer. (Actually, it is easy to 
show $\left|\int_{1}^{x}\left(\delta-\varepsilon t^{-\varepsilon} d t\right)^{\rho}\right|=O(x)$; see the proof of Lemma 4.5.) By (2.1), we have

$$
\begin{aligned}
\widehat{F}(s) & =1+\sum_{k=1}^{\infty} \frac{\rho(\rho-1) \cdots(\rho-k+1)}{k !}\left(-\varepsilon \int_{1}^{\infty} x^{-s-\varepsilon} d x\right)^{k} \\
& =1+\sum_{k=1}^{\infty} \frac{\rho(\rho-1) \cdots(\rho-k+1)}{k !}\left(\frac{-\varepsilon}{s-(1-\varepsilon)}\right)^{k} \\
& =\exp \left\{\rho \log \left(1-\frac{\varepsilon}{s-(1-\varepsilon)}\right)\right\}=\left(1-\frac{\varepsilon}{s-(1-\varepsilon)}\right)^{\rho},
\end{aligned}
$$

where $\log z=\log |z|+i \arg z$ with $-\pi<\arg z \leq \pi$. Therefore

$$
\int_{1}^{\infty} x^{-s}\left(\delta-\varepsilon x^{-\varepsilon} d x\right)^{\rho}=\frac{(s-1)^{\rho}}{(s-(1-\varepsilon))^{\rho}} \text { for } \sigma>1 \text {. }
$$

Similarly, by (2.3), we have

$$
\int_{1}^{\infty} x^{-s}\left(\delta+(1-\varepsilon) x^{-\varepsilon} d x\right)^{\rho}=\frac{s^{\rho}}{(s-(1-\varepsilon))^{\rho}}
$$

for $\sigma>1-\varepsilon$. Furthermore,

$$
\int_{1}^{\infty} x^{-s} \log x(\delta+d x)^{\rho}=\rho\left(\frac{s^{\rho}}{(s-1)^{\rho+1}}-\frac{s^{\rho-1}}{(s-1)^{\rho}}\right) .
$$

\section{Asymptotic anAlysis}

Instead of $x \sum_{\nu=1}^{n} A_{\nu} \log ^{\rho_{\nu}-1} x$ on the right-hand side of (1.3) (or (1.4)), we shall use $\sum_{\mu=1}^{m} B_{\mu} \int_{1}^{x}(\delta+d t)^{\tau_{\mu}}$ which is easy to deal with by using convolution techniques. We first show that the latter is a good approximation of the former by using Beurling's idea [2].

Lemma 3.1. Let $\rho>0,0 \leq \varepsilon<1$. If $\rho=r$ is an integer, then, for $x>1$,

$$
\begin{aligned}
\int_{1}^{x}(\delta & \left.+(1-\varepsilon) t^{-\varepsilon} d t\right)^{r}=\frac{(1-\varepsilon)^{r-1} x^{1-\varepsilon}(\log x)^{r-1}}{\Gamma(r)} \\
& +\sum_{k=1}^{r-1} \frac{(r-1)(r-2) \cdots(r-k)}{k ! \Gamma(r-k)}(1-\varepsilon)^{r-k-1} x^{1-\varepsilon}(\log x)^{r-k-1} .
\end{aligned}
$$

If $\rho$ is not an integer, then, for any positive integer $m$,

$$
\begin{aligned}
\int_{1}^{x} & (\delta \\
& \left.+(1-\varepsilon) t^{-\varepsilon} d t\right)^{\rho}=\frac{(1-\varepsilon)^{\rho-1} x^{1-\varepsilon}(\log x)^{\rho-1}}{\Gamma(\rho)} \\
& +\sum_{k=1}^{m-1} \frac{(\rho-1)(\rho-2) \cdots(\rho-k)}{k ! \Gamma(\rho-k)}(1-\varepsilon)^{\rho-k-1} x^{1-\varepsilon}(\log x)^{\rho-k-1} \\
& +O_{m}\left(x^{1-\varepsilon}(\log x)^{\rho-m-1}\right)
\end{aligned}
$$

holds as $x \rightarrow \infty$, where the $O_{m}$-constant is uniform for $\varepsilon$ satisfying $0 \leq \varepsilon \leq \varepsilon_{0}$ for each fixed $\varepsilon_{0}<1$.

Proof. It suffices to prove (3.1) and (3.2) with $m \geq \rho$. The method of our proof is standard. We consider the Mellin transform

$$
\widehat{F}(s)=\int_{1}^{\infty} x^{-s}\left(\delta+(1-\varepsilon) x^{-\varepsilon} d x\right)^{\rho}=\frac{s^{\rho}}{(s-1+\varepsilon)^{\rho}}
$$


for $\sigma>1-\varepsilon$. Therefore, for $x>1$,

$$
\int_{1}^{x}\left(\delta+(1-\varepsilon) t^{-\varepsilon} d t\right)^{\rho}=\frac{1}{2 \pi i} \int_{\sigma=\sigma_{0}} \frac{x^{s} s^{\rho-1}}{(s-1+\varepsilon)^{\rho}} d s
$$

where $\sigma_{0}>1-\varepsilon$, by Perron's inversion formula.

In the case $\rho=r$, the right-hand side of (3.3) equals

$$
\begin{aligned}
& \sum_{k=0}^{r-1}\left(\begin{array}{c}
r-1 \\
k
\end{array}\right)(1-\varepsilon)^{r-k-1} \frac{1}{2 \pi i} \int_{\sigma=\sigma_{0}} \frac{x^{s}}{(s-1+\varepsilon)^{r-k}} d s \\
& \quad=\sum_{k=0}^{r-1}\left(\begin{array}{c}
r-1 \\
k
\end{array}\right)(1-\varepsilon)^{r-k-1} \frac{1}{\Gamma(r-k)} x^{1-\varepsilon}(\log x)^{r-k-1}
\end{aligned}
$$

and (3.1) follows.

Hence, in the sequel, we assume that $\rho$ is not an integer. As usual, we can shift the integration contour of the integral on the right-hand side of (3.3) to a loop, denoted by $l_{\eta}(1-\varepsilon)$, which consists of the half-line on the lower edge of the real axis from $-\infty$ to $1-\varepsilon-\eta$, the circle $C_{\eta}(1-\varepsilon)$, cut at the point $s=1-\varepsilon-\eta$, with center $s=1-\varepsilon$ and radius $\eta$ sufficiently small, and the half-line on the upper edge of the real axis from $1-\varepsilon-\eta$ to $-\infty$. Thus we have

$$
\int_{1}^{x}\left(\delta+(1-\varepsilon) t^{-\varepsilon} d t\right)^{\rho}=\frac{1}{2 \pi i} \int_{l_{\eta}(1-\varepsilon)} \frac{x^{s} s^{\rho-1}}{(s-1+\varepsilon)^{\rho}} d s .
$$

Let $\varepsilon_{0}$ be fixed and $0 \leq \varepsilon_{0}<1$. Assume $0 \leq \varepsilon \leq \varepsilon_{0}$. Let $\alpha=\frac{1}{2}\left(1-\varepsilon_{0}\right)$. Let $0<\eta<\alpha$ and $l_{\eta}^{\prime}(1-\varepsilon)=l_{\eta}(1-\varepsilon) \cap\{|s-1+\varepsilon| \leq 1-\varepsilon-\alpha\}$. Let $l_{\eta}^{\prime \prime}(1-\varepsilon)$ denote the remaining part of $l_{\eta}(1-\varepsilon)$. Then it is easy to see that

$$
\int_{l_{\eta}^{\prime \prime}(1-\varepsilon)} \frac{x^{s} s^{\rho-1}}{(s-1+\varepsilon)^{\rho}} d s=O\left(x^{\alpha} / \log x\right)
$$

where, and thereafter without repeat, the 0 -constant depends only on $\varepsilon_{0}$ and is uniform for $\varepsilon$ satisfying $0 \leq \varepsilon \leq \varepsilon_{0}$. In the disk $\{|s-1+\varepsilon| \leq 1-\varepsilon-\alpha\}$, we have

$s^{\rho-1}=(1-\varepsilon)^{\rho-1}+\sum_{k=1}^{m-1} \frac{(\rho-1)(\rho-2) \cdots(\rho-k)}{k !}(1-\varepsilon)^{\rho-k-1}(s-1+\varepsilon)^{k}+R(s)$

where

Therefore,

$$
R(s)=O\left(|s-1+\varepsilon|^{m}\right) .
$$

$$
\begin{aligned}
\frac{1}{2 \pi i} \int_{l_{\eta}^{\prime}(1-\varepsilon)} & \frac{x^{s} s^{\rho-1}}{(s-1+\varepsilon)^{\rho}} d s=\frac{(1-\varepsilon)^{\rho-1}}{2 \pi i} \int_{l_{\eta}^{\prime}(1-\varepsilon)} \frac{x^{s}}{(s-1+\varepsilon)^{\rho}} d s \\
& +\sum_{k=1}^{m-1} \frac{(\rho-1)(\rho-2) \cdots(\rho-k)}{k !}(1-\varepsilon)^{\rho-k-1} \\
& \cdot \frac{1}{2 \pi i} \int_{l_{\eta}^{\prime}(1-\varepsilon)} x^{s}(s-1+\varepsilon)^{k-\rho} d s \\
& +\frac{1}{2 \pi i} \int_{l_{\eta}^{\prime}(1-\varepsilon)} \frac{x^{s} R(s)}{(s-1+\varepsilon)^{\rho}} d s .
\end{aligned}
$$


We denote by $l_{\eta}(0)$ and $l_{\eta}^{\prime}(0)$ the respective translations of $l_{\eta}(1-\varepsilon)$ and $l_{\eta}^{\prime}(1-\varepsilon)$ via $w=s-1+\varepsilon$. It is easy to see that

$$
\begin{aligned}
& \frac{1}{2 \pi i} \int_{l_{\eta}^{\prime}(1-\varepsilon)} \frac{x^{s}}{(s-1+\varepsilon)^{\rho}} d s=\frac{x^{1-\varepsilon}}{2 \pi i} \int_{l_{\eta}^{\prime}(0)} e^{w \log x} w^{-\rho} d w \\
& \quad=x^{1-\varepsilon} \log ^{\rho-1} x\left(\frac{1}{2 \pi i} \int_{l_{\eta}^{\prime}(0)} e^{w} w^{-\rho} d w+O\left(\frac{x^{-1+\varepsilon+\alpha}}{\log ^{\rho} x}\right)\right) \\
& \quad=\frac{x^{1-\varepsilon} \log ^{\rho-1} x}{\Gamma(\rho)}+O\left(x^{\alpha} \log ^{-1} x\right) .
\end{aligned}
$$

In the same way we have

$$
\frac{1}{2 \pi i} \int_{l_{\eta}^{\prime}(1-\varepsilon)} x^{s}(s-1+\varepsilon)^{k-\rho} d s=\frac{x^{1-\varepsilon}(\log x)^{\rho-k-1}}{\Gamma(\rho-k)}+O\left(x^{\alpha} \log ^{-1} x\right) .
$$

Finally, we have

$$
\begin{aligned}
\int_{l_{\eta}^{\prime}(1-\varepsilon)} \frac{x^{s} R(s)}{(s-1+\varepsilon)} d s & =O\left(\int_{\alpha}^{1-\varepsilon} x^{\sigma}|\sigma-1+\varepsilon|^{m-\rho} d \sigma\right) \\
& =O\left(x^{1-\varepsilon}(\log x)^{\rho-m-1}\right) .
\end{aligned}
$$

Now, (3.2) follows from (3.4) to (3.9).

Lemma 3.2. Let $\rho>0,0<\varepsilon \leq 1$. If $\rho=r$ is an integer, then

$$
\begin{aligned}
& \int_{1}^{x}\left(\delta-\varepsilon t^{-\varepsilon} d t\right)^{\rho} \\
& \quad=x^{1-\varepsilon} \sum_{n=0}^{r-1} \frac{(-1)^{n-1}}{n !}\left(\sum_{k=n+1}^{r}\left(\begin{array}{l}
r \\
k
\end{array}\right) \frac{\varepsilon^{k}}{(1-\varepsilon)^{k-n}}\right) \log ^{n} x+\frac{1}{(1-\varepsilon)^{r}} .
\end{aligned}
$$

If $\rho$ is not an integer, then, for any positive integer $m$,

$$
\begin{aligned}
& \int_{1}^{x}\left(\delta-\varepsilon t^{-\varepsilon} d t\right)^{\rho} \\
&=x \sum_{n=0}^{m-1}(-1)^{n-1} \pi^{-1} \sin \pi(\rho+n) \Gamma(\rho+n+1) \\
& \cdot\left(\sum_{k=0}^{n} \frac{\rho(\rho+1) \cdots(\rho+k-1)}{k !} \varepsilon^{-k-\rho}\right)(\log x)^{-\rho-n-1} \\
&+O_{m}\left(x(\log x)^{-\rho-m-1}\right)
\end{aligned}
$$

holds as $x \rightarrow \infty$ (where the $O_{m}$-constant is uniform for $\varepsilon$ satisfying $\varepsilon_{0} \leq \varepsilon \leq 1$ for each fuxed $\varepsilon_{0}>0$ ).

Proof. The proof is very close to the one of Lemma 3.1 and hence a sketch of it will be sufficient. The Mellin transform

$$
\widehat{F}(s):=\int_{1}^{\infty} x^{-s}\left(\delta-\varepsilon x^{-\varepsilon} d x\right)^{\rho}
$$

is convergent in the half-plane $\operatorname{Re} s=\sigma>1-\varepsilon$ if $\rho=r$ is an integer. Hence,

$$
\widehat{F}(s)=\left(1-\frac{\varepsilon}{s-1+\varepsilon}\right)^{r}=\frac{(s-1)^{r}}{(s-1+\varepsilon)^{r}} .
$$


Therefore, by Perron's inversion formula,

$$
\begin{aligned}
\int_{1}^{x}( & \left.\delta-\varepsilon t^{-\varepsilon} d t\right)^{\rho}=\frac{1}{2 \pi i} \int_{\sigma=\sigma_{0}} \frac{x^{s}}{s} \frac{(s-1)^{r}}{(s-1+\varepsilon)^{r}} d s \\
= & \sum_{k=0}^{r}\left(\begin{array}{l}
r \\
k
\end{array}\right)(-\varepsilon)^{k} \frac{1}{2 \pi i} \int_{\sigma=\sigma_{0}} \frac{x^{s}}{s} \frac{1}{(s-1+\varepsilon)^{k}} d s \\
= & \sum_{k=0}^{r}\left(\begin{array}{l}
r \\
k
\end{array}\right)(-\varepsilon)^{k}\left(x^{1-\varepsilon} \sum_{\substack{m+n=k-1 \\
m, n \geq 0}} \frac{(-1)^{m} \log ^{n} x}{(1-\varepsilon)^{m+1} n !}+\frac{(-1)^{k}}{(1-\varepsilon)^{k}}\right),
\end{aligned}
$$

where $\sigma_{0}>1-\varepsilon$, and (3.10) follows.

If $\rho$ is not an integer, then $\widehat{F}(s)$ is convergent in the half-plane $\sigma>1$ and (3.12) holds for $\sigma>1$. Therefore, we have

$$
\int_{1}^{x}\left(\delta-\varepsilon t^{-\varepsilon} d t\right)^{\rho}=\frac{1}{2 \pi i} \int_{\sigma=\sigma_{0}} \frac{x^{s}}{s} \frac{(s-1)^{\rho}}{(s-1+\varepsilon)^{\rho}} d s
$$

where $\sigma_{0}>1$. Then we shift the integration contour to a new one, denoted by $l_{\eta}(1)$ with $0<\eta<\varepsilon_{0} / 4$, which consists of the half-lines on the lower edge of the real axis from $-\infty$ to $-\eta$ and on the upper edge from $-\eta$ to $-\infty$, the circle $C$, cut at points $s=-\eta$ and $s=1-\varepsilon+\eta$, with center $s=\frac{1}{2}(1-\varepsilon)$ and radius $\frac{1}{2}(1-\varepsilon)+\eta$, the two line segments on the lower edge of the real axis from $1-\varepsilon+\eta$ to $1-\eta$ and on the upper edge from $1-\eta$ to $1-\varepsilon+\eta$, and the small circle $c_{\eta}(1)$, cut at the point $s=1-\eta$, with center $s=1$ and radius $\eta$. Let $l_{\eta}^{\prime}(1)=l_{\eta}(1) \cap\left\{|s-1| \leq \varepsilon_{0} / 2\right\}$. Then, it is easy to see that

$$
\begin{aligned}
\int_{1}^{x}\left(\delta-\varepsilon t^{-\varepsilon} d t\right)^{\rho}=\frac{1}{2 \pi i} \int_{l_{\eta}(1)} \frac{x^{s}}{s} \frac{(s-1)^{\rho}}{(s-1+\varepsilon)^{\rho}} d s \\
=\frac{1}{2 \pi i} \int_{l_{\eta}^{\prime}(1)} \frac{x^{s}}{s} \frac{(s-1)^{\rho}}{(s-1+\varepsilon)^{\rho}} d s+O_{\varepsilon_{0}}\left(x^{1-\varepsilon_{0} / 2} \log ^{-1} x\right)
\end{aligned}
$$

and

$$
\begin{aligned}
\frac{1}{2 \pi i} \int_{l_{\eta}^{\prime}(1)} \frac{x^{s}}{s} \frac{(s-1)^{\rho}}{(s-1+\varepsilon)^{\rho}} d s \\
=\sum_{n=0}^{m}(-1)^{n}\left(\sum_{k=0}^{n} \frac{\rho(\rho+1) \cdots(\rho+k-1)}{k !} \varepsilon^{-k-\rho)}\right. \\
\quad \cdot \frac{1}{2 \pi i} \int_{l_{\eta}^{\prime}(1)} x^{s}(s-1)^{\rho+n} d s \\
\quad+\frac{1}{2 \pi i} \int_{l_{\eta}^{\prime}(1)} x^{s}(s-1)^{\rho} R(s) d s
\end{aligned}
$$

where

$$
R(s)=O_{m, \varepsilon_{0}}\left(|s-1|^{m+1}\right) .
$$

Evaluating the integrals on the right-hand side of (3.14) as we did in the proof of Lemma 3.1, we arrive at (3.11).

The following lemma is the main result of this section. 
Lemma 3.3. Let $0<\rho_{1}<\rho_{2}<\cdots<\rho_{n}$. Then there exist $0<\tau_{1}<\tau_{2}<\cdots<$ $\tau_{m}$ with $\tau_{m}=\rho_{n}$ and $\tau_{\mu}=\rho_{\nu}-k$ for some nonnegative integer $k$ such that, as $x \rightarrow \infty$,

$$
x \sum_{\nu=1}^{n} A_{\nu}(\log x)^{\rho_{\nu}-1}=\sum_{\mu=1}^{m} B_{\mu} \int_{1}^{x}(\delta+d t)^{\tau_{\mu}}+R(x),
$$

where $B_{m}=A_{n} \Gamma\left(\rho_{n}\right)$ and $R(x)=O\left(x(\log x)^{-2+\alpha}\right)$ with $\alpha=\max \left\{\rho_{\nu}-\left[\rho_{\nu}\right]\right.$, $\nu=1,2, \ldots, n\}$ if $\rho_{\nu}, \nu=1,2, \ldots, n$, are not all integers and $R(x)=0$ otherwise.

Proof. Without loss of generality, we may assume $n=1$. We shall prove (3.15) by induction. We first consider the case that $\rho_{1}$ is not an integer. For $0<\rho_{1}<1$, we have, by (3.2) with $\varepsilon=0, m=1$, and $\rho=\rho_{1}$,

$$
A_{1} x(\log x)^{\rho_{1}-1}=A_{1} \Gamma\left(\rho_{1}\right) \int_{1}^{x}(\delta+d t)^{\rho_{1}}+O\left(x(\log x)^{-2+\rho_{1}}\right)
$$

and (3.15) is true. Then, for $k<\rho_{1}<k+1$ with $k \geq 1$, by (3.2) with $\varepsilon=0$, $m=\left[\rho_{1}\right]+1$, and $\rho=\rho_{1}$, we have

$$
\begin{aligned}
A_{1} x(\log x)^{\rho_{1}-1}= & A_{1} \Gamma\left(\rho_{1}\right) \int_{1}^{x}(\delta+d t)^{\rho_{1}} \\
& -\sum_{\mu=1}^{\left[\rho_{1}\right]} \bar{B}_{\mu} x(\log x)^{\rho_{1}-\mu-1}+O\left(x(\log x)^{-2+\rho_{1}-\left[\rho_{1}\right]}\right) .
\end{aligned}
$$

Note that, for $\mu=1,2, \ldots,\left[\rho_{1}\right], \rho_{1}-\mu$ are all nonintegers, $\rho_{1}-\mu<k$, and $\rho_{1}-\mu-\left[\rho_{1}-\mu\right]=\rho_{1}-\left[\rho_{1}\right]$. Therefore, by hypothesis of induction, we have

$$
\begin{aligned}
A_{1} x(\log x)^{\rho_{1}-1}= & A_{1} \Gamma\left(\rho_{1}\right) \int_{1}^{x}(\delta+d t)^{\rho_{1}} \\
& +\sum_{\mu=1}^{\left[\rho_{1}\right]} B_{\mu} \int_{1}^{x}(\delta+d t)^{\tau_{\mu}}+O\left(x(\log x)^{-2+\rho_{1}-\left[\rho_{1}\right]}\right)
\end{aligned}
$$

and (3.15) holds.

Similarly, by using (3.1), we can prove the truth of (3.15) when $\rho_{1}$ is an integer.

\section{APPROXimate CONVOlution INVERSE}

Let

$$
P(x)=\sum_{\mu=1}^{m} B_{\mu} \int_{1}^{x}(\delta+d t)^{\tau_{\mu}} \text { for } x \geq 1
$$

which is defined in (3.15) of Lemma 3.3, and $P(x)=0$ for $x<1$. Define a function $Q_{1}(x)$ as follows. If $\tau_{m}$ and $\tau_{m}-\tau_{\mu}, \mu=1,2, \ldots, m-1$, are all integers, let

$$
Q_{1}(x)=\max \left\{\sup _{x \leq y} \frac{|N(y)-P(y)|}{y}, x^{-1 / 2}\right\}
$$


If $\tau_{m}-\tau_{\mu}, \mu=1,2, \ldots, m-1$, are all integers but $\tau_{m}$ is not, let

$$
Q_{1}(x)=\max \left\{\sup _{x \leq y} \frac{|N(y)-P(y)|}{y},(\log e x)^{-1-\tau_{m}+\left[\tau_{m}\right]}\right\} .
$$

If $\tau_{m}$ is an integer but $\tau_{m}-\tau_{\mu}, \mu=1,2, \ldots, m-1$, are not all integers, let $(4.2)_{3} \quad Q_{1}(x)=\max \left\{\sup _{x \leq y} \frac{|N(y)-P(y)|}{y},(\log e x)^{-1-\beta},(\log e x)^{-3 / 2+\gamma / 2}\right\}$,

where

$$
\beta=\min \left\{\tau_{m}-\tau_{\mu}-k: \tau_{m}-k-1<\tau_{\mu}<\tau_{m}-k, 1 \leq \mu \leq m-1\right\}
$$

and

(4.4) $\gamma=\max \left\{\tau_{\mu}-\tau_{m}+k+1: \tau_{m}-k-1<\tau_{\mu}<\tau_{m}-k, 1 \leq \mu \leq m-1\right\}$

so that $0<\beta<1,0<\gamma<1$. Finally, if $\tau_{m}$ is not an integer and $\tau_{m}-\tau_{\mu}$, $\mu=1,2, \ldots, m-1$, are not all integers, let

$$
\begin{array}{r}
Q_{1}(x)=\max \left\{\sup _{x \leq y} \frac{|N(y)-P(y)|}{y},(\log e x)^{-1-\tau_{m}+\left[\tau_{m}\right]},\right. \\
\left.(\log e x)^{-1-\beta},(\log e x)^{-3 / 2+\gamma / 2}\right\} .
\end{array}
$$

Lemma 4.1. Let $Q_{1}(x)$ be the function defined in (4.2). Assume (1.4). Then there exists a function $Q(x)$ defined for $x \geq 0$ such that

$$
\begin{gathered}
Q(x) \text { is nonincreasing; } \\
\int_{1}^{\infty} Q(x) x^{-1} d x<\infty ; \\
Q(x) \leq 4 Q\left(x^{2}\right) \quad \text { for all } x \geq 1 ; \\
Q(x) \geq Q_{1}(x) .
\end{gathered}
$$

Moreover,

$$
Q(x)=o\left(\log ^{-1} e x\right)
$$

$$
\begin{aligned}
& \text { for } x \geq 1, \\
& \int_{1}^{x} x t^{-1} Q(x / t) Q(t) d t \leq C_{1} \min \left\{x Q(x), \int_{1}^{x} Q(t) d t\right\},
\end{aligned}
$$

where $C_{1}$ is a constant.

Proof. By Lemma 3.3, we have

$$
\sup _{x \leq y} \frac{|N(y)-P(y)|}{y} \leq \sup _{x \leq y} y^{-1}\left|N(y)-\sum_{\nu=1}^{n} A_{\nu}(\log y)^{\rho_{\nu}-1}\right|+R(x)
$$

where $R(x)=O\left((\log x)^{-2+\alpha}\right)$ with $\alpha=\max \left\{\rho_{\nu}-\left[\rho_{\nu}\right]\right\}$ if $\rho_{\nu}, \nu=1, \ldots, n$, are not all integers and $R(x)=0$ otherwise. Therefore, by (1.4), (4.2), and (4.11),

$$
\int_{1}^{\infty} Q_{1}(x) x^{-1} d x<\infty
$$


Moreover, $Q_{1}(x)$ is nonnegative and nonincreasing. Define $Q(x)$ recursively by setting

$$
Q(x)=\left\{\begin{array}{l}
Q_{1}(1) \text { for } 0 \leq x<2, \\
\max \left\{Q_{1}\left(2^{2^{m-1}}\right), 4^{-1} Q\left(2^{2^{m-1}}\right)\right\} \text { for } 2^{2^{m-1}}<x \leq 2^{2^{m}}, m \in \mathbf{N} .
\end{array}\right.
$$

Then we can verify that this function satisfies condition (4.5) to (4.9) as we did in the proof of Lemmas 1 and 2 of [8].

Moreover, from Lemma 3 of [8], we have, for $x \geq 1$,

$$
\int_{1}^{x} x t^{-1} Q(x / t) Q(t) d t \leq C_{1}^{\prime} x Q(x) \text {. }
$$

If $x \leq 2$, then

If $x>2$, then, by (4.12),

$$
\int_{1}^{x} x t^{-1} Q(x / t) Q(t) d t \leq C_{2}^{\prime} \int_{1}^{x} Q(t) d t .
$$

$$
\int_{1}^{x} x t^{-1} Q(x / t) Q(t) d t \leq C_{1}^{\prime} x Q(x) \leq 2 C_{1}^{\prime} \int_{1}^{x} Q(t) d t
$$

since $Q(x)$ is nonincreasing. This proves (4.10).

Lemma 4.2. Let $0<\rho<1, \varepsilon>0$, and $T>1$. Let

$$
\alpha(x)=\alpha_{\rho}(x)= \begin{cases}\frac{x(\log e x)^{\rho-1}}{\Gamma(\rho)}, & \text { if } x \geq T, \\ 0, & \text { if } x<T .\end{cases}
$$

Then, for $x>T$,

$$
\begin{gathered}
\left|\int_{1}^{x} d \alpha(t) *\left(\delta-\varepsilon t^{-\varepsilon} d t\right)\right| \leq \frac{T^{\varepsilon}}{(\log e T)^{(1-\rho) / 2} \Gamma(\rho)} \\
\cdot\left\{x^{1-\varepsilon}+\int_{1}^{x}\left(\frac{x}{t}\right)^{1-\varepsilon}(\log e t)^{-3 / 2+\rho / 2} d t\right\} .
\end{gathered}
$$

Proof. We have

$$
\begin{aligned}
I(x) & :=\int_{1}^{x} d \alpha(t) *\left(\delta-\varepsilon t^{-\varepsilon} d t\right) \\
& =\alpha(x)-\varepsilon x^{1-\varepsilon} \int_{1}^{x} \alpha(t) t^{-2+\varepsilon} d t
\end{aligned}
$$

and

$$
\begin{aligned}
\int_{1}^{x} \alpha(t) t^{-2+\varepsilon} d t & =\frac{1}{\Gamma(\rho)} \int_{T}^{x} t^{-1+\varepsilon}(\log e t)^{\rho-1} d t \\
= & \frac{1}{\varepsilon \Gamma(\rho)}\left\{x^{\varepsilon}(\log e x)^{\rho-1}-T^{\varepsilon}(\log e T)^{\rho-1}\right. \\
& \left.+(1-\rho) \int_{T}^{x} t^{-1+\varepsilon}(\log e t)^{\rho-2} d t\right\}
\end{aligned}
$$

by integration by parts. Hence

$$
\begin{aligned}
|I(x)| & =\frac{1}{\Gamma(\rho)}\left|\frac{T^{\varepsilon} x^{1-\varepsilon}}{(\log e T)^{1-\rho}}-(1-\rho) \int_{T}^{x}\left(\frac{x}{t}\right)^{1-\varepsilon}(\log e t)^{\rho-2} d t\right| \\
& \leq \frac{T^{\varepsilon}}{\Gamma(\rho)(\log e T)^{(1-\rho) / 2}}\left\{x^{1-\varepsilon}+\int_{1}^{x}\left(\frac{x}{t}\right)^{1-\varepsilon}(\log e t)^{-3 / 2+\rho / 2} d t\right\} .
\end{aligned}
$$


Lemma 4.3. Let $0<\tau_{1}<\tau_{2}<\cdots<\tau_{m}$ and $0<\varepsilon \leq 1 / 2$. Assume $\tau_{m}=\tau \geq 1$. If $\tau-\tau_{\mu}$ is a positive integer then

$$
\begin{aligned}
& \left|\int_{1}^{x}(\delta+d t)^{\tau_{\mu}} *\left(\delta-\varepsilon t^{-\varepsilon} d t\right)^{\tau}\right| \\
& \quad \leq \int_{1}^{x}\left(\delta+(1-\varepsilon) t^{-\varepsilon} d t\right)^{\tau-1}+2 \varepsilon \int_{1}^{x}\left(\delta+(1-\varepsilon) t^{-\varepsilon} d t\right)^{\tau} .
\end{aligned}
$$

If $\tau-\tau_{\mu}$ is not an integer and $\tau_{\mu}>\tau-[\tau]$, then for any given $T>1$, there exists a constant $C_{2}$ dependent on $T$ but independent of $\varepsilon$ for which (4.15)

$$
\begin{aligned}
\mid \int_{1}^{x}(\delta & +d t)^{\tau_{\mu}} *\left(\delta-\varepsilon t^{-\varepsilon} d t\right)^{\tau} \mid \\
\leq & C_{2}\left\{\int_{1}^{x} \frac{x}{t} Q(x / t)\left(\delta+(1-\varepsilon) t^{-\varepsilon} d t\right)^{\tau-1}+\int_{1}^{x}\left(\delta+(1-\varepsilon) t^{-\varepsilon} d t\right)^{\tau-1}\right\} \\
& +2 \varepsilon C_{2}\left\{\int_{1}^{x} x t^{-1} Q(x / t)\left(\delta+(1-\varepsilon) t^{-\varepsilon} d t\right)^{\tau}+\int_{1}^{x}\left(\delta+(1-\varepsilon) t^{-\varepsilon} d t\right)^{\tau}\right\} \\
& +\frac{T^{\varepsilon}}{C_{3}(\log e T)^{(1-\gamma) / 2}}\left\{\int_{1}^{x}\left(\delta+(1-\varepsilon) t^{-\varepsilon} d t\right)^{\tau}\right. \\
& \left.+\int_{1}^{x}\left(\delta+(1-\varepsilon) t^{-\varepsilon} d t\right)^{\tau} *(\log e t)^{-3 / 2+\gamma / 2} d t\right\},
\end{aligned}
$$

where $Q(x)$ and $\gamma$ are defined in Lemma 4.1 and in (4.4) respectively, $C_{3}=$ $\min \left\{\Gamma\left(\tau_{\mu}-\tau+k+1\right): \tau-k-1<\tau_{\mu}<\tau-k, 1 \leq \mu \leq m-1\right\}$.

Proof. If $\tau-\tau_{\mu}$ is a positive integer, $\tau_{\mu}=\tau-k-1$. Then we have, by (2.6),

$$
\begin{aligned}
\mid \int_{1}^{x}(\delta & +d t)^{\tau_{\mu}} *\left(\delta-\varepsilon t^{-\varepsilon} d t\right)^{\tau} \mid \\
= & \left|\int_{1}^{x}\left(\delta+(1-\varepsilon) t^{-\varepsilon} d t\right)^{\tau-k-1} *\left(\delta-\varepsilon t^{-\varepsilon} d t\right)^{k+1}\right| \\
\leq & \int_{1}^{x}\left(\delta+(1-\varepsilon) t^{-\varepsilon} d t\right)^{\tau-k-1} \\
& *\left(\delta+2 \varepsilon \sum_{l=1}^{k+1}\left(\begin{array}{c}
k+1 \\
l
\end{array}\right)(1-\varepsilon)^{l} \frac{\log ^{l-1} t}{(l-1) !} t^{-\varepsilon} d t\right) \\
\leq & \int_{1}^{x}\left(\delta+(1-\varepsilon) t^{-\varepsilon} d t\right)^{\tau-k-1}+2 \varepsilon \int_{1}^{x}\left(\delta+(1-\varepsilon) t^{-\varepsilon} d t\right)^{\tau} .
\end{aligned}
$$

This proves (4.14).

If $\tau-\tau_{\mu}$ is not an integer and $\tau_{\mu}>\tau-[\tau]$, then there exists a nonnegative integer $k$ such that $0 \leq \tau-k-1<\tau_{\mu}<\tau-k$ since $\tau \geq 1$. Let

$$
\alpha(x)= \begin{cases}\frac{x(\log e x)^{\tau_{\mu}-\tau+k}}{\Gamma\left(\tau_{\mu}-\tau+k+1\right)} & \text { for } x \geq T, \\ 0 & \text { for } x<T .\end{cases}
$$


Then we have

$$
\begin{aligned}
\int_{1}^{x}(\delta & +d t)^{\tau_{\mu}} *\left(\delta-\varepsilon t^{-\varepsilon} d t\right)^{\tau} \\
= & \int_{1}^{x}\left(\delta+(1-\varepsilon) t^{-\varepsilon} d t\right)^{\tau-k-1} * d \alpha(t) *\left(\delta-\varepsilon t^{-\varepsilon} d t\right)^{k+1} \\
& +\int_{1}^{x}\left(\delta+(1-\varepsilon) t^{-\varepsilon} d t\right)^{\tau-k-1} \\
& *\left(d \frac{t(\log e t)^{\tau_{\mu}-\tau+k}}{\Gamma\left(\tau_{\mu}-\tau+k+1\right)}-d \alpha(t)\right) *\left(\delta-\varepsilon t^{-\varepsilon} d t\right)^{k+1} \\
& +\int_{1}^{x}\left(\delta+(1-\varepsilon) t^{-\varepsilon} d t\right)^{\tau-k-1} \\
& *\left((\delta+d t)^{\tau_{\mu}-\tau+k+1}-d \frac{t(\log e t)^{\tau_{\mu}-\tau+k}}{\Gamma\left(\tau_{\mu}-\tau+k+1\right)}\right) \\
& *\left(\delta-\varepsilon t^{-\varepsilon} d t\right)^{k+1} \\
= & \mathrm{I}_{1}+\mathrm{I}_{2}+\mathrm{I}_{3},
\end{aligned}
$$

say. By Lemma 3.1 with $\varepsilon=0$ and Lemma 4.1,

$$
\begin{array}{r}
\left|\int_{1}^{x}(\delta+d t)^{\tau_{\mu}-\tau+k+1}-\frac{x(\log e x)^{\tau_{\mu}-\tau+k}}{\Gamma\left(\tau_{\mu}-\tau+k+1\right)}\right| \\
\leq C_{1}^{\prime} x(\log x)^{\tau_{\mu}-\tau+k-1} \leq C_{2}^{\prime} x Q(x),
\end{array}
$$

since $\tau-\tau_{\mu}-k \geq \beta$, where $\beta$ is defined in (4.3). Therefore,

$$
\begin{aligned}
\left|\mathrm{I}_{3}\right| \leq & C_{2}^{\prime} \int_{1}^{x} x t^{-1} Q(x / t)\left(\delta+(1-\varepsilon) t^{-\varepsilon} d t\right)^{\tau-k-1} *\left(\delta+\varepsilon t^{-\varepsilon} d t\right)^{k+1} \\
\leq & C_{2}^{\prime} \int_{1}^{x} x t^{-1} Q(x / t)\left(\delta+(1-\varepsilon) t^{-\varepsilon} d t\right)^{\tau-k-1} \\
& +2 \varepsilon C_{2}^{\prime} \int_{1}^{x} x t^{-1} Q(x / t)\left(\delta+(1-\varepsilon) t^{-\varepsilon} d t\right)^{\tau}
\end{aligned}
$$

Plainly, $\left(\delta+(1-\varepsilon) t^{-\varepsilon} d t\right)^{\tau-k-1}$ is a nonnegative measure since $\tau-k-1 \geq 0$ and we have

$$
\begin{aligned}
\left|\mathrm{I}_{2}\right| \leq & C_{3}^{\prime} \int_{1}^{x}\left(\delta+(1-\varepsilon) t^{-\varepsilon} d t\right)^{\tau-k-1} *\left(\delta+\varepsilon t^{-\varepsilon} d t\right)^{k+1} \\
\leq & C_{3}^{\prime} \int_{1}^{x}\left(\delta+(1-\varepsilon) t^{-\varepsilon} d t\right)^{\tau-k-1} \\
& +2 \varepsilon C_{3}^{\prime} \int_{1}^{x}\left(\delta+(1-\varepsilon) t^{-\varepsilon} d t\right)^{\tau}
\end{aligned}
$$

where the constant $C_{3}^{\prime}$ depends only on $T$. Finally, by Lemma 4.2 with $\rho=$ 
$\tau_{\mu}-\tau+k+1$

$$
\begin{aligned}
\left|\mathrm{I}_{1}\right|= & \left|\int_{1}^{x}\left(\int_{1}^{x / t} d \alpha(u) *\left(\delta-\varepsilon u^{-\varepsilon} d u\right)\right)\left(\left(\delta+(1-\varepsilon) t^{-\varepsilon} d t\right)^{\tau-k-1} *\left(\delta-\varepsilon t^{-\varepsilon} d t\right)^{k}\right)\right| \\
\leq & \frac{T^{\varepsilon}}{C_{3}(\log e T)^{(1-\gamma) / 2}} \int_{1}^{x}\left\{\left(\frac{x}{t}\right)^{1-\varepsilon}+\int_{1}^{x / t}\left(\delta+(1-\varepsilon) u^{-\varepsilon} d u\right) *(\log e u)^{-3 / 2+\gamma / 2} d u\right\} \\
& \cdot\left(\delta+(1-\varepsilon) t^{-\varepsilon} d t\right)^{\tau-k-1} *\left(\delta+\varepsilon t^{-\varepsilon} d t\right)^{k} \\
\leq & \frac{T^{\varepsilon}}{C_{3}(\log e T)^{(1-\gamma) / 2}}\left\{\int_{1}^{x}\left(\delta+(1-\varepsilon) t^{-\varepsilon} d t\right)^{\tau}\right. \\
& \left.\quad \int_{1}^{x}\left(\delta+(1-\varepsilon) t^{-\varepsilon} d t\right)^{\tau} *(\log e t)^{-3 / 2+\gamma / 2} d t\right\},
\end{aligned}
$$

since $\tau_{\mu}-\tau+k+1<\gamma$, where $\gamma$ is defined in (4.4). Now, from (4.16) to (4.19), (4.15) follows.

Lemma 4.4. Let $0<\rho<1$. Then

$$
\int_{1}^{x}\left|\left(\delta-t^{-1} d t\right)^{\rho}\right|=O\left(x(\log x)^{-\rho-1}\right) .
$$

Proof. We have

$$
\begin{aligned}
\left(\delta-t^{-1} d t\right)^{\rho} & =\delta+\sum_{k=1}^{\infty} \frac{\rho(\rho-1)(\rho-2) \cdots(\rho-k+1)}{k !}(-1)^{k} t^{-1} \frac{(\log t)^{k-1}}{(k-1) !} d t \\
& =\delta-\left\{\rho+\sum_{k=2}^{\infty} \frac{\rho(1-\rho)(2-\rho) \cdots(k-\rho-1)}{k !} \frac{\log ^{k-1} t}{(k-1) !}\right\} t^{-1} d t \\
& =\delta-f(t) t^{-1} d t,
\end{aligned}
$$

say. Then $f(t) \geq 0$. Therefore,

$$
\int_{1}^{x}\left(\delta-t^{-1} d t\right)^{\rho}=1-\int_{1}^{x} f(t) t^{-1} d t
$$

and hence

$$
\begin{aligned}
\int_{1}^{x}\left|\left(\delta-t^{-1} d t\right)^{\rho}\right| & =1+\int_{1}^{x} f(t) t^{-1} d t=2-\int_{1}^{x}\left(\delta-t^{-1} d t\right)^{\rho} \\
& =O\left(x(\log x)^{-\rho-1}\right)
\end{aligned}
$$

by (3.11) with $\varepsilon=1$.

The establishment of the following lemma is a main step in the proof of the Theorem.

Lemma 4.5. Assume $\tau=\tau_{m} \geq 1$ and (4.5), (4.6), (4.7), (4.8). Then, for fixed and sufficiently small $\varepsilon>0$, we have

$$
U_{\varepsilon}(x):=\int_{1}^{x} d N *\left(\delta-\varepsilon t^{-\varepsilon} d t\right)^{\tau} * Q(t) d t \geq 0
$$

for all $x \geq 1$ and $U_{\varepsilon}(x) \rightarrow \infty$ as $x \rightarrow \infty$.

Proof. We have

$$
\left(\delta-\varepsilon t^{-\varepsilon} d t\right)^{\tau}=\delta+t^{-\varepsilon} f(t) d t
$$


where

$$
f(t)=\sum_{k=1}^{\infty} \frac{\tau(\tau-1) \cdots(\tau-k+1)}{k !}(-\varepsilon)^{k} \frac{(\log t)^{k-1}}{(k-1) !} .
$$

Since $\tau>0$, there exists a constant $K$ such that

$$
\left|\frac{\tau(\tau-1) \cdots(\tau-k+1)}{k !}\right| \leq K
$$

holds for $k=1,2, \ldots$. Therefore, we have

$$
t^{-\varepsilon}|f(t)| \leq K \varepsilon t^{-\varepsilon} \sum_{k=1}^{\infty} \frac{(\varepsilon \log t)^{k-1}}{(k-1) !}=K \varepsilon .
$$

Hence,

$$
\begin{aligned}
\int_{1}^{x} d N *\left(\delta-\varepsilon t^{-\varepsilon} d t\right)^{\tau}=\int_{1}^{x} d N *\left(\delta+t^{-\varepsilon} f(t) d t\right) \\
\quad \geq N(x)-\int_{1}^{x} d N * K \varepsilon d t \geq N(x)-K \varepsilon N(x)(x-1) \geq 0
\end{aligned}
$$

for $1 \leq x \leq(K \varepsilon)^{-1}$. The lemma is certainly true for $1 \leq x \leq(K \varepsilon)^{-1}$, since the third convolution factor is everywhere nonnegative.

Therefore, we assume $x \geq(K \varepsilon)^{-1}$ and $\varepsilon<1 / 2$ and shall utilize all the convolution factors. We write, by (2.4),

$$
\begin{aligned}
U_{\varepsilon}(x)= & A \int_{1}^{x}\left(\delta+(1-\varepsilon) t^{-\varepsilon} d t\right)^{\tau} * Q(t) d t \\
& +\sum_{\mu=1}^{m-1} B_{\mu} \int_{1}^{x}(\delta+d t)^{\tau_{\mu}} *\left(\delta-\varepsilon t^{-\varepsilon} d t\right)^{\tau} * Q(t) d t \\
& +\int_{1}^{x}\left(d N-\sum_{\mu=1}^{m} B_{\mu}(\delta+d t)^{\tau_{\mu}}\right) *\left(\delta-\varepsilon t^{-\varepsilon} d t\right)^{\tau} * Q(t) d t \\
= & \mathrm{I}_{1}+\mathrm{I}_{2}+\mathrm{I}_{3},
\end{aligned}
$$

say, where $A=A_{n} \Gamma\left(\rho_{n}\right)>0$ and $\sum_{\mu=1}^{m} B_{\mu} \int_{1}^{x}(\delta+d t)^{\tau_{\mu}}$ is defined in (3.15). We shall show that $I_{1}$ is positive, that $I_{1} \rightarrow \infty$ as $x \rightarrow \infty$, and that $I_{2}$ and $I_{3}$ are negligible. Actually, we have

$$
\begin{aligned}
\mathrm{I}_{1} & \geq A \int_{1}^{x}\left(\delta+(1-\varepsilon) t^{-\varepsilon} d t\right) * Q(t) d t=A \int_{1}^{x}\left(\frac{x}{t}\right)^{1-\varepsilon} Q(t) d t \\
& \geq A x^{1-\varepsilon} \int_{1}^{x} t^{-1} Q(t) d t \rightarrow \infty \quad \text { as } x \rightarrow \infty
\end{aligned}
$$

We then estimate $\left|I_{2}\right|$ and $\left|I_{3}\right|$. By (4.8),

$$
\left|\int_{1}^{x}\left(d N-\sum_{\mu=1}^{m} B_{\mu}(\delta+d t)^{\tau_{\mu}}\right) * Q(t) d t\right| \leq \int_{1}^{x} x t^{-1} Q(x / t) Q(t) d t .
$$


If $\tau$ is an integer, we have

$$
\begin{aligned}
\left|\mathbf{I}_{3}\right| & \leq \int_{1}^{x}\left|\int_{1}^{x / t}\left(d N-\sum_{\mu=1}^{m} B_{\mu}(\delta+d u)^{\tau_{\mu}}\right) * Q(u) d u\right|\left(\delta+\varepsilon t^{-\varepsilon} d t\right)^{\tau} \\
& \leq C_{1} x Q(x)+2 C_{1} \varepsilon \int_{1}^{x}\left(\delta+(1-\varepsilon) t^{-\varepsilon} d t\right)^{\tau} * Q(t) d t
\end{aligned}
$$

by (4.22) and (4.10). If $\tau$ is not an integer, then $\tau>1$, and we have

$$
\begin{aligned}
(d N & \left.-\sum_{\mu=1}^{m} B_{\mu}(\delta+d t)^{\tau_{\mu}}\right) *\left(\delta-\varepsilon t^{-\varepsilon} d t\right)^{\tau} * Q(t) d t \\
= & \left(d N-\sum_{\mu=1}^{m} B_{\mu}(\delta+d t)^{\tau_{\mu}}\right) *\left(\delta+(1-\varepsilon) t^{-\varepsilon} d t\right)^{\tau-[\tau]} \\
& *\left(\delta-t^{-1} d t\right)^{\tau-[\tau]} *\left(\delta-\varepsilon t^{-\varepsilon} d t\right)^{[\tau]} * Q(t) d t
\end{aligned}
$$

since

$$
\left(\delta-\varepsilon t^{-\varepsilon} d t\right)^{\tau-[\tau]}=\left(\delta+(1-\varepsilon) t^{-\varepsilon} d t\right)^{\tau-[\tau]} *\left(\delta-t^{-1} d t\right)^{\tau-[\tau]} .
$$

Therefore,

$$
\begin{aligned}
&\left|\mathrm{I}_{3}\right| \leq \int_{1}^{x}\left|\int_{1}^{x / t}\left(d N-\sum_{\mu=1}^{m} B_{\mu}(\delta+d u)^{\tau_{\mu}}\right) * Q(u) d u\right| \\
& \leq C_{1} \int_{1}^{x}\left(\int_{1}^{x / t} Q(u) d u\right)\left(\left(\delta+(1-\varepsilon) t^{-\varepsilon} d t\right)^{\tau-[\tau]} *\left|\left(\delta-t^{-1} d t\right)^{\tau-[\tau]}\right| *\left(\delta+\varepsilon t^{-\varepsilon} d t\right)^{[\tau]}\right) \\
&\left.*\left|\left(\delta-t^{-1} d t\right)^{\tau-[\tau]}\right| *\left(\delta+\varepsilon t^{-\varepsilon} d t\right)^{[\tau]}\right) \\
&=C_{1} \int_{1}^{x} Q(t) d t *\left|\left(\delta-t^{-1} d t\right)^{\tau-[\tau]}\right| \\
& *\left(\delta+(1-\varepsilon) t^{-\varepsilon} d t\right)^{\tau-[\tau]} *\left(\delta+\varepsilon t^{-\varepsilon} d t\right)^{[\tau]}
\end{aligned}
$$

by (4.22) and (4.10). Then, by Lemma 4.4 with $\rho=\tau-[\tau]$ and Lemma 4.1,

$$
\begin{aligned}
\int_{1}^{x}\left|\left(\delta-t^{-1} d t\right)^{\tau-[\tau]}\right| * Q(t) d t & \leq C_{4} \int_{1}^{x} x t^{-1} Q(x / t) Q(t) d t \\
& \leq C_{1} C_{4} \int_{1}^{x} Q(t) d t
\end{aligned}
$$

It follows that

$$
\begin{aligned}
\left|\mathbf{I}_{3}\right| \leq C_{1}^{2} C_{4}\left\{\int_{1}^{x}\right. & (\delta \\
& \left.+(1-\varepsilon) t^{-\varepsilon} d t\right)^{\tau-[\tau]} * Q(t) d t \\
& \left.+2 \varepsilon \int_{1}^{x}\left(\delta+(1-\varepsilon) t^{-\varepsilon} d t\right)^{\tau} * Q(t) d t\right\} .
\end{aligned}
$$


To evaluate $\left|I_{2}\right|$, we note that, by (4.10),

$$
\begin{gathered}
\int_{1}^{x}\left(\int_{1}^{x / t} \frac{x}{u t} Q\left(\frac{x}{u t}\right)\left(\delta+(1-\varepsilon) u^{-\varepsilon} d u\right)^{\tau-1}\right) Q(t) d t \\
\quad=\int_{1}^{x} d(t Q(t)) *\left(\delta+(1-\varepsilon) t^{-\varepsilon} d t\right)^{\tau-1} * Q(t) d t \\
\quad \leq C_{1} \int_{1}^{x}\left(\delta+(1-\varepsilon) t^{-\varepsilon} d t\right)^{\tau-1} * Q(t) d t
\end{gathered}
$$

and

$$
\begin{gathered}
\int_{1}^{x}\left(\int_{1}^{x / t} \frac{x}{u t} Q\left(\frac{x}{u t}\right)\left(\delta+(1-\varepsilon) u^{-\varepsilon} d u\right)^{\tau}\right) Q(t) d t \\
\leq C_{1} \int_{1}^{x}\left(\delta+(1-\varepsilon) t^{-\varepsilon} d t\right)^{\tau} * Q(t) d t .
\end{gathered}
$$

Let $C_{5}$ be a constant such that

$$
\int_{1}^{x}(\log e t)^{-3 / 2+\gamma / 2} d t \leq C_{5} x(\log e x)^{-3 / 2+\gamma / 2} \text { for all } x \geq 1,
$$

where $\gamma$ is defined in (4.4). Then

$$
\int_{1}^{x}(\log e t)^{-3 / 2+\gamma / 2} d t \leq C_{5} x Q(x) \text { for all } x \geq 1
$$

by (4.8). Hence,

$$
\begin{aligned}
\int_{1}^{x}(\log e t)^{-3 / 2+\gamma / 2} d t * Q(t) d t & \leq C_{5} \int_{1}^{x} x t^{-1} Q(x / t) Q(t) d t \\
& \leq C_{1} C_{5} \int_{1}^{x} Q(t) d t .
\end{aligned}
$$

by (4.10). Also, we note that if $\tau_{\mu}<\tau-[\tau]$ then, by an analogue of (4.24),

$$
\begin{aligned}
& \left|\int_{1}^{x}(\delta+d t)^{\tau_{\mu}} *\left(\delta-\varepsilon t^{-\varepsilon} d t\right)^{\tau} * Q(t) d t\right| \\
& \leq \int_{1}^{x}\left(\delta+(1-\varepsilon) t^{-\varepsilon} d t\right)^{\tau-[\tau]} *\left|\left(\delta-t^{-1} d t\right)^{\tau-[\tau]-\tau_{\mu}}\right| \\
& *\left(\delta+\varepsilon t^{-\varepsilon} d t\right)^{[\tau]} * Q(t) d t \\
& \leq C_{1} C_{4} \int_{1}^{x}\left(\delta+(1-\varepsilon) t^{-\varepsilon} d t\right)^{\tau-[\tau]} * Q(t) d t *\left(\delta+\varepsilon t^{-\varepsilon} d t\right)^{[\tau]} \\
& \leq C_{1} C_{4}\left\{\int_{1}^{x}\left(\delta+(1-\varepsilon) t^{-\varepsilon} d t\right)^{\tau-[\tau]} * Q(t) d t\right. \\
& \left.+2 \varepsilon \int_{1}^{x}\left(\delta+(1-\varepsilon) t^{-\varepsilon} d t\right)^{\tau} * Q(t) d t\right\} .
\end{aligned}
$$


Therefore, by (4.14), (4.15), (4.26), (4.27), (4.28), and (4.29), we have

$$
\begin{array}{r}
\left|\mathrm{I}_{2}\right| \leq \sum_{\mu=1}^{m-1}\left|B_{\mu}\right|\left\{\int_{1}^{x}\left(\delta+(1-\varepsilon) t^{-\varepsilon} d t\right)^{\tau-1} * Q(t) d t\right. \\
\left.\quad+2 \varepsilon \int_{1}^{x}\left(\delta+(1-\varepsilon) t^{-\varepsilon} d t\right)^{\tau} * Q(t) d t\right\} \\
+\sum_{\mu=1}^{m-1}\left|B_{\mu}\right|\left\{C_{2}\left(C_{1}+1\right) \int_{1}^{x}\left(\delta+(1-\varepsilon) t^{-\varepsilon} d t\right)^{\tau-1} * Q(t) d t\right. \\
+2 \varepsilon C_{2}\left(C_{1}+1\right) \int_{1}^{x}\left(\delta+(1-\varepsilon) t^{-\varepsilon} d t\right)^{\tau} * Q(t) d t \\
+\frac{T^{\varepsilon}\left(1+C_{1} C_{5}\right)}{\left.C_{3}(\log e T)^{(1-\gamma) / 2} \int_{1}^{x}\left(\delta+(1-\varepsilon) t^{-\varepsilon} d t\right)^{\tau} * Q(t) d t\right\}} \\
+\sum_{\mu=1}^{m-1}\left|B_{\mu}\right| C_{1} C_{4}\left\{\int_{1}^{x}\left(\delta+(1-\varepsilon) t^{-\varepsilon}\right)^{\tau-[\tau]} * Q(t) d t\right. \\
\left.+2 \varepsilon \int_{1}^{x}\left(\delta+(1-\varepsilon) t^{-\varepsilon} d t\right)^{\tau} * Q(t) d t\right\},
\end{array}
$$

where

$$
\sum_{\mu=1}^{m-1}, \quad \sum_{\mu=1}^{m-1}, \text { and } \sum_{\mu=1}^{m-1}
$$

denote the respective sums over all $\tau_{\mu}$ such that $\tau-\tau_{\mu}$ is an integer, such that $\tau-\tau_{\mu}$ is not an integer but $\tau_{\mu}>\tau-[\tau]$, and such that $\tau_{\mu}<\tau-[\tau]$.

We now choose a number $T$ sufficiently large so that

$$
\frac{\sum_{\mu=1}^{\prime \prime m-1}\left|B_{\mu}\right|\left(1+C_{1} C_{5}\right)}{C_{3}(\log e T)^{(1-\gamma) / 2}}<\frac{A}{16}
$$

and fix it. If $\tau$ is an integer, we can choose a positive number $\varepsilon_{0}$ sufficiently small so that $\left(K \varepsilon_{0}\right)^{-1}>T, T^{\varepsilon_{0}}<2$ and that

$$
2 \varepsilon_{0}\left(\sum_{\mu=1}^{m-1}\left|B_{\mu}\right|+C_{2}\left(C_{1}+1\right) \sum_{\mu=1}^{m-1}\left|B_{\mu}\right|+C_{1} C_{4} \sum_{\mu=1}^{m-1}\left|B_{\mu}\right|+C_{1}\right)<\frac{A}{8}
$$

Then, for $\varepsilon \leq \varepsilon_{0}$, from (4.21), (4.23), (4.30), (4.31), and (4.32), we have

$$
\begin{aligned}
U_{\varepsilon}(x) \geq & \frac{3 A}{4} \int_{1}^{x}\left(\delta+(1-\varepsilon) t^{-\varepsilon} d t\right)^{\tau} * Q(t) d t \\
& -C_{6} \int_{1}^{x}\left(\delta+(1-\varepsilon) t^{-\varepsilon} d t\right)^{\tau-1} * Q(t) d t-C_{1} x Q(x)
\end{aligned}
$$

for $x \geq(K \varepsilon)^{-1}$, where

$$
C_{6}=\sum_{\mu=1}^{m-1}\left|B_{\mu}\right|+C_{2}\left(C_{1}+1\right) \sum_{\mu=1}^{m-1}\left|B_{\mu}\right|+C_{1} C_{4} \sum_{\mu=1}^{m-1}\left|B_{\mu}\right| .
$$


By (3.1), there exists a number $x_{0}$ sufficiently large and independent of $\varepsilon$ such that

$$
\frac{A}{4} \int_{1}^{x}\left(\delta+(1-\varepsilon) t^{-\varepsilon} d t\right)^{\tau} \geq C_{6} \int_{1}^{x}\left(\delta+(1-\varepsilon) t^{-\varepsilon} d t\right)^{\tau-1}
$$

holds for $x \geq x_{0}$ and all $\varepsilon$ satisfying $0<\varepsilon \leq \varepsilon_{0}$. Hence, from (4.33), for $\varepsilon \leq\left(K x_{0}^{2}\right)^{-1}\left(\leq \varepsilon_{0}\right)$ and $x>(K \varepsilon)^{-1}$, we have

$$
\begin{aligned}
U_{\varepsilon}(x) \geq & \frac{A}{2} \int_{1}^{x}\left(\delta+(1-\varepsilon) t^{-\varepsilon} d t\right)^{\tau} * Q(t) d t \\
& -C_{6} \int_{1}^{x_{0}}\left(\delta+(1-\varepsilon) t^{-\varepsilon} d t\right)^{\tau-1} \int_{x / x_{0}}^{x} Q(t) d t-C_{1} x Q(x) \\
\geq & \frac{A}{2} \int_{1}^{x}\left(\delta+(1-\varepsilon) t^{-\varepsilon} d t\right)^{\tau} * Q(t) d t \\
& -\left(4 C_{6} \int_{1}^{x_{0}}\left(\delta+(1-\varepsilon) t^{-\varepsilon} d t\right)^{\tau-1}+C_{1}\right) x Q(x)
\end{aligned}
$$

since $x \geq x_{0}^{2}$ and

$$
\int_{x / x_{0}}^{x} Q(t) d t \leq Q\left(x / x_{0}\right) x \leq Q(\sqrt{x}) x \leq 4 x Q(x)
$$

by (4.5) and (4.7). Now, for sufficiently small $\varepsilon>0$,

$$
\begin{aligned}
& \frac{A}{4} \int_{1}^{x}\left(\delta+(1-\varepsilon) t^{-\varepsilon} d t\right)^{\tau} * Q(t) d t \\
& \quad \geq \frac{A}{4} \int_{1}^{x}\left(\delta+(1-\varepsilon) t^{-\varepsilon} d t\right) * Q(t) d t \\
& \quad=\frac{A}{4} x \int_{1}^{x} t^{-1-\varepsilon} Q(x / t) d t \geq \frac{A}{4} x Q(x) \int_{1}^{1 / K \varepsilon} t^{-1-\varepsilon} d t \\
& \quad \geq \frac{A}{4} x Q(x)(K \varepsilon)^{\varepsilon} \log \frac{1}{K \varepsilon} \\
& \quad \geq\left(4 C_{6} \int_{1}^{x_{0}}\left(\delta+(1-\varepsilon) t^{-\varepsilon} d t\right)^{\tau-1}+C_{1}\right) x Q(x)
\end{aligned}
$$

since $(K \varepsilon)^{\varepsilon} \geq \varepsilon^{\varepsilon} \geq \exp \left(-e^{-1}\right)$. Therefore, $U_{\varepsilon}(x) \geq \frac{1}{4} \mathrm{I}_{1}$ for $x \geq(K \varepsilon)^{-1}$.

If $\tau$ is not an integer, we choose a positive number $\varepsilon_{0}$ sufficiently small so that

$$
2 \varepsilon_{0}\left(\sum_{\mu=1}^{m-1}\left|B_{\mu}\right|+C_{2}\left(C_{1}+1\right) \sum_{\mu=1}^{m-1}\left|B_{\mu}\right|+C_{1} C_{4} \sum_{\mu=1}^{m-1}\left|B_{\mu}\right|+C_{1}^{2} C_{4}\right)<\frac{A}{8}
$$

Then, from (4.25), (4.24), (4.30), (4.31), and (4.34), we have

$$
\begin{aligned}
U_{\varepsilon}(x) \geq & \frac{3 A}{4} \int_{1}^{x}\left(\delta+(1-\varepsilon) t^{-\varepsilon} d t\right)^{\tau} * Q(t) d t \\
& -C_{6} \int_{1}^{x}\left(\delta+(1-\varepsilon) t^{-\varepsilon} d t\right)^{\tau-1} * Q(t) d t \\
& -C_{1}^{2} C_{4} \int_{1}^{x}\left(\delta+(1-\varepsilon) t^{-\varepsilon} d t\right)^{\tau-[\tau]} * Q(t) d t .
\end{aligned}
$$


In the same way as above, by (3.2),

$$
\begin{aligned}
\frac{A}{4} \int_{1}^{x}(\delta+ & \left.(1-\varepsilon) t^{-\varepsilon} d t\right)^{\tau} \geq C_{6} \int_{1}^{x}\left(\delta+(1-\varepsilon) t^{-\varepsilon} d t\right)^{\tau-1} \\
& +C_{1}^{2} C_{4} \int_{1}^{x}\left(\delta+(1-\varepsilon) t^{-\varepsilon} d t\right)^{\tau-[\tau]}
\end{aligned}
$$

holds for $x \geq x_{0}$ and all $\varepsilon$ satisfying $0<\varepsilon \leq \varepsilon_{0}$ and hence, from (4.35),

$$
\begin{aligned}
U_{\varepsilon}(x) \geq & \frac{A}{2} \int_{1}^{x}\left(\delta+(1-\varepsilon) t^{-\varepsilon} d t\right)^{\tau} * Q(t) d t \\
& -C_{6} \int_{1}^{x_{0}}\left(\delta+(1-\varepsilon) t^{-\varepsilon} d t\right)^{\tau-1} \int_{x / x_{0}}^{x} Q(t) d t \\
& -C_{1}^{2} C_{4} \int_{1}^{x_{0}}\left(\delta+(1-\varepsilon) t^{-\varepsilon} d t\right)^{\tau-[\tau]} \int_{x / x_{0}}^{x} Q(t) d t \\
\geq & \frac{A}{2} \int_{1}^{x}\left(\delta+(1-\varepsilon) t^{-\varepsilon} d t\right)^{\tau} * Q(t) d t \\
& -4\left(C_{6} \int_{1}^{x_{0}}\left(\delta+(1-\varepsilon) t^{-\varepsilon} d t\right)^{\tau-1}\right. \\
& \left.\quad+C_{1}^{2} C_{4} \int_{1}^{x_{0}}\left(\delta+(1-\varepsilon) t^{-\varepsilon} d t\right)^{\tau-[\tau]}\right) x Q(x) .
\end{aligned}
$$

Therefore, for sufficiently small $\varepsilon>0, U_{\varepsilon}(x)>\frac{1}{4} \mathrm{I}_{1}$ for $x \geq(K \varepsilon)^{-1}$.

\section{THE PROOF OF THE THEOREM}

The proof of the Theorem follows the general idea in $[5,8]$.

Lemma 5.1. Suppose that

$$
N(x)=x \sum_{\nu=1}^{n} A_{\nu}(\log x)^{\rho_{\nu}-1}+O\left(x \log ^{-1} e x\right) .
$$

Then we have

$$
\int_{1}^{x} L d N *\left(\delta-\varepsilon t^{-\varepsilon} d t\right)^{\tau}=O_{\varepsilon}(x),
$$

where $\tau=\rho_{n} \geq 1$.

Proof. We have

$$
\begin{array}{rl}
\int_{1}^{x} & L d N *\left(\delta-\varepsilon t^{-\varepsilon} d t\right)^{\tau} \\
& =\int_{1}^{x} L d P *\left(\delta-\varepsilon t^{-\varepsilon} d t\right)^{\tau}+\int_{1}^{x}(L d N-L d P) *\left(\delta-\varepsilon t^{-\varepsilon} d t\right)^{\tau} \\
& =\mathrm{I}_{1}+\mathrm{I}_{2}
\end{array}
$$

say, where $P(x)$ is defined in (4.1). If suffices to show that both $I_{1}$ and $I_{2}$ are $O(x)$. 
Actually, from (5.1) and (3.15), $N(x)-P(x)=O\left(x \log ^{-1} e x\right)$. Therefore, we have

$$
\begin{aligned}
\int_{1}^{x}(L d N-L d P) & =\log x(N(x)-P(x))-\int_{1}^{x}(N(t)-P(t)) t^{-1} d t \\
& =O(x) .
\end{aligned}
$$

If $\tau=r$ is an integer, then plainly,

$$
\begin{aligned}
\mathrm{I}_{2} & =O\left(\int_{1}^{x} x t^{-1}\left(\delta+\varepsilon t^{-\varepsilon} d t\right)^{r}\right) \\
& =O\left(x\left(1+\sum_{k=1}^{r} \varepsilon^{k} \frac{1}{(k-1) !} \int_{1}^{x} t^{-1-\varepsilon}(\log t)^{k-1} d t\right)\right) \\
& =O(x) .
\end{aligned}
$$

If $\tau$ is not an integer, then $0<\tau-[\tau]<1$ and we have

$$
\mathbf{I}_{2}=\int_{1}^{x}(L d N-L d P) *\left(\delta-\varepsilon t^{-\varepsilon} d t\right)^{\tau-[\tau]} *\left(\delta-\varepsilon t^{-\varepsilon} d t\right)^{[\tau]}
$$

and, by Lemma 4.4 ,

$$
\begin{aligned}
& \left|\int_{1}^{x}(L d N-L d P) *\left(\delta-\varepsilon t^{-\varepsilon} d t\right)^{\tau-[\tau]}\right| \\
& \quad \ll \int_{1}^{x} x t^{-1}\left|\left(\delta-\varepsilon t^{-\varepsilon} d t\right)^{\tau-[\tau]}\right| \\
& \quad=x\left\{x^{-1} \int_{1}^{x}\left|\left(\delta-\varepsilon t^{-\varepsilon} d t\right)^{\tau-[\tau]}\right|+\int_{1}^{x}\left(\int_{1}^{t}\left|\left(\delta-\varepsilon u^{-\varepsilon} d u\right)^{\tau-[\tau]}\right|\right) t^{-2} d t\right\} \\
& \quad \ll x
\end{aligned}
$$

by integration by parts. Therefore, (5.3) is still true.

To estimate $I_{1}$, we consider the Mellin transform

$$
\begin{gathered}
\int_{1}^{\infty} x^{-s} L d P *\left(\delta-\varepsilon x^{-\varepsilon} d x\right)^{\tau}=\tau B_{m}\left(\frac{s^{\tau}}{(s-1)(s-1+\varepsilon)^{\tau}}-\frac{s^{\tau-1}}{(s-1+\varepsilon)^{\tau}}\right) \\
+\sum_{\mu=1}^{m-1} \tau_{\mu} B_{\mu}\left(\frac{s^{\tau_{\mu}}(s-1)^{\tau-\tau_{\mu}-1}}{(s-1+\varepsilon)^{\tau}}-\frac{s^{\tau_{\mu}-1}(s-1)^{\tau-\tau_{\mu}}}{(s-1+\varepsilon)^{\tau}}\right)
\end{gathered}
$$

for $\sigma>1$. Then, by Perron's inversion formula,

$$
\begin{aligned}
\mathrm{I}_{1}= & \int_{1}^{x} L d P *\left(\delta-\varepsilon t^{-\varepsilon} d t\right)^{\tau} \\
= & \frac{\tau B_{m}}{2 \pi i}\left(\int_{\sigma=\sigma_{0}} \frac{x^{s}}{s} \frac{s^{\tau}}{(s-1)(s-1+\varepsilon)^{\tau}} d s-\int_{\sigma=\sigma_{0}} \frac{x^{s}}{s} \frac{s^{\tau-1}}{(s-1+\varepsilon)^{\tau}} d s\right) \\
& +\sum_{\mu=1}^{m-1} \tau_{\mu} \frac{B_{\mu}}{2 \pi i}\left(\int_{\sigma=\sigma_{0}} \frac{x^{s}}{s} \frac{s^{\tau}(s-1)^{\tau-\tau_{\mu}-1}}{(s-1+\varepsilon)^{\tau}} d s-\int_{\sigma=\sigma_{0}} \frac{x^{s}}{s} \frac{s^{\tau_{\mu}-1}(s-1)^{\tau-\tau}}{(s-1+\varepsilon)^{\tau}} d s\right),
\end{aligned}
$$


where $\sigma_{0}>1$. As we did in the proof of Lemma 3.2, we shift the integration contour to $l_{\eta}(1)$ and obtain

$$
\frac{1}{2 \pi i} \int_{\sigma=\sigma_{0}} \frac{x^{s}}{s} \frac{s^{\tau}}{(s-1)(s-1+\varepsilon)^{\tau}} d s=\frac{x}{\varepsilon^{\tau}}(1+o(1)),
$$

and

$$
\begin{aligned}
& \frac{1}{2 \pi i} \int_{\sigma=\sigma_{0}} \frac{x^{s}}{s} \frac{s^{\tau_{\mu}-1}(s-1)^{\tau-\tau_{\mu}}}{(s-1+\varepsilon)^{\tau}} d s=O\left(x(\log x)^{-\tau+\tau_{\mu}-1}\right), \\
& \frac{1}{2 \pi i} \int_{\sigma=\sigma_{0}} \frac{x^{s}}{s} \frac{s^{\tau_{\mu}}(s-1)^{\tau-\tau_{\mu}-1}}{(s-1+\varepsilon)^{\tau}} d s=O\left(x(\log x)^{-\tau+\tau_{\mu}}\right)
\end{aligned}
$$

if $\tau-\tau_{\mu}$ is not an integer. Also, as in the proof of Lemma 3.1, we obtain

$$
\begin{aligned}
& \frac{1}{2 \pi i} \int_{\sigma=\sigma_{0}} \frac{x^{s}}{s} \frac{s^{\tau_{\mu}-1}(s-1)^{\tau-\tau_{\mu}}}{(s-1+\varepsilon)^{\tau}} d s=O\left(x^{1-\varepsilon}(\log x)^{\tau-1}\right), \\
& \frac{1}{2 \pi i} \int_{\sigma=\sigma_{0}} \frac{x^{s}}{s} \frac{s^{\tau_{\mu}}(s-1)^{\tau-\tau_{\mu}-1}}{(s-1+\varepsilon)^{\tau}} d s=O\left(x^{1-\varepsilon}(\log x)^{\tau-1}\right),
\end{aligned}
$$

if $\tau-\tau_{\mu}$ is an integer, and

$$
\frac{1}{2 \pi i} \int_{\sigma=\sigma_{0}} \frac{x^{s}}{s} \frac{s^{\tau}-1}{(s-1+\varepsilon)^{\tau}} d s=O\left(x^{1-\varepsilon}(\log x)^{\tau-1}\right)
$$

Therefore,

$$
\mathbf{I}_{1}=\frac{x}{\varepsilon^{\tau}}(1+o(1))=O(x)
$$

We are now in the position to set up the upper estimate of the Theorem. The starting point is the identity $d \psi * d N=L d N$, an analogue of Chebyshev's one. We convolve each side of it by $\left(\delta-\varepsilon t^{-\varepsilon} d t\right)^{\tau} * Q(t) d t$, where $\tau=\tau_{m}=\rho_{n}$, and obtain

$$
\begin{aligned}
\int_{1}^{x} d N * & \left(\delta-\varepsilon t^{-\varepsilon} d t\right)^{\tau} * Q(t) d t * d \psi \\
& =\int_{1}^{x} L d N *\left(\delta-\varepsilon t^{-\varepsilon} d t\right)^{\tau} * Q(t) d t .
\end{aligned}
$$

Proof of the upper estimate. Assume (1.4), then (4.5), (4.6), (4.7), and (4.8) hold by Lemma 4.1. By Lemmas 4.5 and 5.1,

$$
\psi(x / B) \leq \int_{1}^{x} U_{\varepsilon}(x / t) d \psi(t)=O(x)
$$

since $U_{\varepsilon}(x) \geq 1$ for $x \geq B$.

To establish the lower estimate of the Theorem, we need one more lemma.

Lemma 5.2. Suppose that

$$
N(x)=x \sum_{\nu=1}^{n} A_{\nu}(\log x)^{\rho_{\nu}-1}+o\left(x \log ^{-1} e x\right) .
$$

Then we have

$$
\int_{1}^{x} L d N *\left(\delta-t^{-1} d t\right)^{\tau}=A x+o(x)
$$

where $\tau=\rho_{n} \geq 1$ and $A=\tau A_{n} \Gamma(\tau)>0$. 
Proof. As in the proof of Lemma 5.1, the integral on the right-hand side of (5.5) equals

$$
\int_{1}^{x} L d P *\left(\delta-t^{-1} d t\right)^{\tau}+\int_{1}^{x}(L d N-L d P) *\left(\delta-t^{-1} d t\right)^{\tau}=\mathrm{I}_{1}+\mathrm{I}_{2},
$$

say. From (5.4) and (3.15), $N(x)-P(x)=o\left(x \log ^{-1} e x\right)$ and hence

$$
\int_{1}^{x}(L d N-L d P)=o(x) \text {. }
$$

It follows that

$$
\begin{aligned}
\left|\int_{1}^{x}(L d N-L d P) *\left(\delta-t^{-1} d t\right)^{\tau-[\tau]}\right| \\
\leq \varepsilon \int_{1}^{x / M} x t^{-1}\left|\left(\delta-t^{-1} d t\right)^{\tau-[\tau]}\right| \\
\quad+K \int_{x / M}^{x} x t^{-1}\left|\left(\delta-t^{-1} d t\right)^{\tau-[\tau]}\right| \\
\leq \varepsilon(C+1) x
\end{aligned}
$$

for $x$ sufficiently large, since $C=\int_{1}^{\infty} t^{-1}\left|\left(\delta-t^{-1} d t\right)^{\tau-[\tau]}\right|$ is convergent by (4.20) with $\rho=\tau-[\tau]$. Hence

$$
\mathrm{I}_{2}=\int_{1}^{x}(L d N-L d P) *\left(\delta-t^{-1} d t\right)^{\tau-[\tau]} *\left(\delta-t^{-1} d t\right)^{[\tau]}=o(x) .
$$

To estimate $I_{1}$, we consider the Mellin transform

$$
\begin{aligned}
& \int_{1}^{x} x^{-s} L d P *\left(\delta-x^{-1} d x\right)^{\tau} \\
& \quad=\tau B_{m}\left(\frac{1}{s-1}-\frac{1}{s}\right)+\sum_{\mu=1}^{m-1} \tau_{\mu} B_{\mu}\left(\frac{(s-1)^{\tau-\tau_{\mu}-1}}{s^{\tau-\tau_{\mu}}}-\frac{(s-1)^{\tau-\tau_{\mu}}}{s^{\tau-\tau_{\mu}+1}}\right)
\end{aligned}
$$

for $\sigma>1$. As we did in the proof of Lemmas 3.2 and 3.1, we have

$$
\frac{1}{2 \pi i} \int_{\sigma=\sigma_{0}} \frac{x^{s}}{s} \frac{(s-1)^{\tau-\tau_{\mu}-1}}{s^{\tau-\tau_{\mu}}} d s=O\left((\log x)^{\tau-\tau_{\mu}}\right) \quad \text { or } \quad O\left(x(\log x)^{-\tau+\tau_{\mu}}\right)
$$

according as $\tau-\tau_{\mu}$ is an integer or not and a similar estimate for

$$
\frac{1}{2 \pi i} \int_{\sigma=\sigma_{0}} \frac{x^{s}}{s} \frac{(s-1)^{\tau-\tau_{\mu}}}{s^{\tau-\tau_{\mu}+1}} d s .
$$

Therefore, by Perron's inversion formula,

$$
\begin{aligned}
\mathrm{I}_{1}= & \int_{1}^{x} L d P *\left(\delta-t^{-1} d t\right)^{\tau} \\
= & \tau B_{m}\left(\int_{\sigma=\sigma_{0}} \frac{x^{s}}{s} \frac{1}{s-1} d s-\int_{\sigma=\sigma_{0}} \frac{x^{s}}{s^{2}} d s\right) \\
& +\sum_{\mu=1}^{m-1} \tau_{\mu} B_{\mu}\left(\int_{\sigma=\sigma_{0}} \frac{x^{s}}{s} \frac{(s-1)^{\tau-\tau_{\mu}-1}}{s^{\tau-\tau_{\mu}}} d s-\int_{\sigma=\sigma_{0}} \frac{x^{s}}{s} \frac{(s-1)^{\tau-\tau_{\mu}}}{s^{\tau-\tau_{\mu}+1}} d s\right) \\
= & \tau B_{m} x+o(x) .
\end{aligned}
$$

Now, (5.5) follows from (5.6) and (5.7). 
Proof of the lower estimate. Assume (1.4). Then from (3.15), (4.8), (4.9), and (4.2), (5.4) is true. Hence, (5.5) holds by Lemma 5.2. Let

$$
\begin{aligned}
U_{1}(x) & :=\int_{1}^{x} d N *\left(\delta-t^{-1} d t\right)^{\tau} \\
& =\int_{1}^{x} d P *\left(\delta-t^{-1} d t\right)^{\tau}+\int_{1}^{x}(d N-d P) *\left(\delta-t^{-1} d t\right)^{\tau} \\
& =\mathrm{I}_{1}+\mathrm{I}_{2},
\end{aligned}
$$

say. We shall first show that both $\mathrm{I}_{1}$ and $\mathrm{I}_{2}$ are $O\left(1+\int_{1}^{x} Q(t) d t\right)$ and hence so is $U_{1}(x)$.

Actually, if $\tau$ is not an integer, we have

$$
\left|\int_{1}^{x}(d N-d P) *\left(\delta-t^{-1} d t\right)^{\tau-[\tau]}\right| \ll \int_{1}^{x} x t^{-1} Q(x / t)\left|\left(\delta-t^{-1} d t\right)^{\tau-[\tau]}\right|
$$

by (4.2) and (4.8). By integration by parts, the right-hand side equals

$$
\begin{aligned}
Q(1) & \int_{1}^{x}\left|\left(\delta-t^{-1} d t\right)^{\tau-[\tau]}\right|-x \int_{1}^{x}\left(\int_{1}^{t}\left|\left(\delta-u^{-1} d u\right)^{\tau-[\tau]}\right|\right) d\left(t^{-1} Q(x / t)\right) \\
\leq & Q(1) \int_{1}^{x}\left|\left(\delta-t^{-1} d t\right)^{\tau-[\tau]}\right| \\
& +x \int_{1}^{x}\left(\int_{1}^{t}\left|\left(\delta-u^{-1} d u\right)^{\tau-[\tau]}\right|\right) t^{-2} Q(x / t) d t
\end{aligned}
$$

since $Q(x / t)$ is nondecreasing in $t$. Therefore we have

$$
\begin{aligned}
& \left|\int_{1}^{x}(d N-d P) *\left(\delta-t^{-1} d t\right)^{\tau-[\tau]}\right| \\
& \quad \ll x(\log e x)^{-\tau+[\tau]-1}+x \int_{1}^{x} t^{-1}(\log e t)^{-\tau+[\tau]-1} Q(x / t) d t \\
& \quad \ll x Q(x)+x \int_{1}^{x} t^{-1} Q(t) Q(x / t) d t \\
& \quad \ll x Q(x),
\end{aligned}
$$

by Lemmas 4.4 and 4.1. If $\tau$ is an integer, (5.9) is plainly true. It follows that

$$
\begin{aligned}
\mathrm{I}_{2} & =\int_{1}^{x}(d N-d P) *\left(\delta-t^{-1} d t\right)^{\tau-[\tau]} *\left(\delta-t^{-1} d t\right)^{[\tau]} \\
& \ll \int_{1}^{x} x t^{-1} Q(x / t)\left(\delta+t^{-1} d t\right)^{[\tau]} \\
& =x Q(x)+x \sum_{k=1}^{[\tau]} \frac{1}{(k-1) !} \int_{1}^{x} t^{-2} Q(x / t) \log ^{k-1} t d t \\
& \ll x Q(x)+\int_{1}^{x} x t^{-1} Q(x / t) Q(t) d t
\end{aligned}
$$


since $t^{-1}(\log t)^{k-1} \ll Q(t)$ by (4.2). Hence,

$$
\mathrm{I}_{2} \ll 1+\int_{1}^{x} Q(t) d t
$$

by (4.10).

To evaluate $\mathrm{I}_{1}$, we have, by Lemma 3.2 with $\rho=\tau-\tau_{\mu}$ and $\varepsilon=1$,

$$
\int_{1}^{x}\left(\delta-t^{-1} d t\right)^{\tau-\tau_{\mu}}=O\left((\log x)^{\tau-\tau_{\mu}-1}\right) \quad \text { or } O\left(x(\log x)^{-\tau+\tau_{\mu}-1}\right)
$$

according as $\tau-\tau_{\mu}$ is an integer or not. It follows that

$$
\begin{aligned}
\mathrm{I}_{1} & =1+\sum_{\mu=1}^{m-1} B_{\mu} \int_{1}^{x}\left(\delta-t^{-1} d t\right)^{\tau-\tau_{\mu}} \\
& \ll x Q(x) \ll 1+\int_{1}^{x} Q(t) d t
\end{aligned}
$$

by (4.8). Now, from (5.8), (5.10), and (5.11),

$$
U_{1}(x) \ll 1+\int_{1}^{x} Q(t) d t .
$$

From the analogue of Chebyshev's identity, we have

$$
\int_{1}^{x} d N *\left(\delta-t^{-1} d t\right)^{\tau} * d \psi=\int_{1}^{x} L d N *\left(\delta-t^{-1} d t\right)^{\tau} .
$$

By (5.5), it turns out that

$$
\int_{1}^{x} U_{1}(x / t) d \psi(t)=A x+o(x) .
$$

We note that, by the upper estimate of $\psi(x)$,

$$
\begin{aligned}
\int_{1}^{x} U_{1}(x / t) d \psi(t) & \leq K \int_{1}^{x}\left(1+\int_{1}^{x / t} Q(u) d u\right) d \psi(t) \\
& \leq K\left\{\psi(x)+\int_{1}^{x} \psi(x / t) Q(t) d t\right\} \\
& \leq K\left\{\psi(x)\left(1+\int_{1}^{B} Q(t) d t\right)+C_{7} x \int_{B}^{x} t^{-1} Q(t) d t\right\} \\
& \leq C_{8} \psi(x)+C_{7} K x \int_{B}^{x} t^{-1} Q(t) d t .
\end{aligned}
$$

For $B$ sufficiently large, $C_{7} K \int_{B}^{x} t^{-1} Q(t) d t \leq \frac{1}{3} A$. Fixing $B$, for $x$ sufficiently large, we have $C_{8} \psi(x) \geq \frac{1}{3} A x$. This completes the proof of the Theorem.

\section{REFERENCES}

1. P. T. Bateman and H. G. Diamond, Asymptomatic distribution of Beurling's generalized prime numbers, Studies in Number Theory, Vol. 6, Math. Assoc. Amer., Prentice-Hall, Englewood Cliffs, N.J., 1969. pp. 152-210.

2. A. Beurling, Analyse de la loi asymptotique de la distribution des nombres premiers généralisés. I, Acta Math. 68 (1937), 225-291. 
3. H. G. Diamond, Asymptotic distribution of Beurling's generalized integers, Illinois J. Math. 14 (1970), 12-28.

4. $\ldots$ A set of generalized numbers showing Beurling's theorem to be sharp, Illinois J. Math. 14 (1970), 29-34.

5. __ Chebyshev estimates for Beurling generalized prime numbers, Proc. Amer. Math. Soc. 39 (1973), 503-508.

6. __, Chebyshev type estimates in prime number theory, Séminaire de Théorie des Nombres, Année 1974-1975 (Univ. Bordeaux I, Talence), exposé $n^{\circ} 24$.

7. R. S. Hall, Beurling generalized prime number system in which the Chebyshev inequalities fail, Proc. Amer. Math. Soc. 40 (1973), 79-82.

8. W.-B. Zhang, Chebyshev type estimates for Beurling generalized prime numbers, Proc. Amer. Math. Soc. 101 (1987), 205-212.

Department of Mathematics, University of Illinois, Urbana, ILlinOIs 61801

E-mail address: wbzhang@symcom.math.uiuv.edu 